\title{
Laurent Cassegrain
}

\author{
Riad Haidar, \\ haidar@onera.fr
}

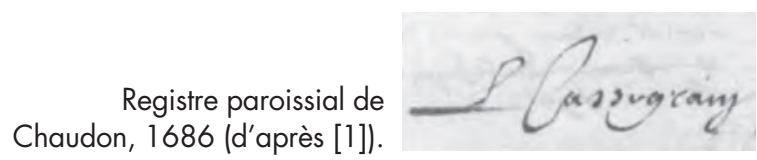

Principales dates

Vers 1629 - Naissance dans la région de Chartres (France)

1654 Professeur au Collège Pocquet de Chartres

1672 Articles sur la trompette à parler de loin, et sur le télescope

1685 Nommé prêtre de la paroisse de Chaudon

31 août 1693 - Mort à Chaudon (France)

Malgré l'immense renommée que lui vaut son concept de télescope, Cassegrain a été durant trois siècles et reste, pour une large part, un parfait inconnu. Grâce au travail d'enquête de deux astronomes français, on sait aujourd'hui que, probablement, il se prénommait Laurent et assumait la charge de curé de la paroisse de Chaudon. Pour l'identifier, point de portait, mais une signature que nous reproduisons ici.

\section{Le mystère Cassegrain}

En avril 1672, le sieur de Bercé, pseudonyme du prieur Claude Etienne, chanoine de Chartres, publie dans le supplément du Journal des Sçavans deux lettres d'un dénommé Cassegrain. La première lettre décrit un prototype de trompettes à parler de loin (le précurseur du mégaphone). La seconde vient en réaction à un article récent sur le télescope qu'lsaac Newton a présenté à la Royal Society : le sieur de Bercé y décrit un schéma de télescope réflectif proposé par Cassegrain, qu'il trouve particulièrement pertinent et qui s'avère sensiblement différent de celui de Newton. Depuis, le concept de Cassegrain s'est largement répandu, au point de devenir un classique pour les astronomes. Comme ses contemporains inventés par James Gregory (en 1663) et Isaac Newton (en 1670), il $s^{\prime}$ inscrit dans la logique des systèmes catadioptriques initiés par Mersenne quelques décennies plus tôt, formés par la combinaison de deux miroirs. Parmi eux, le Gregory est le duo parabole-ellipse (les deux miroirs étant concaves), le Newton est le duo parabole-plan, et enfin le Cassegrain est le duo parabole-hyperbole (le premier étant concave et le second convexe, ce qui lui confère une compacité exceptionnelle). À la différence du télescope Newton, les architectures Gregory et Cassegrain utilisent un miroir primaire percé en son centre, de sorte que les axes optiques des deux miroirs coïncident et que l'image formée peut être perçue par un observateur placé derrière le télescope. Cassegrain est le premier à décrire cet espace d'observation, et il lui doit certainement sa notoriété persistante. En effet, bien que sa combinaison ne soit plus guère utilisée en astronomie et ait cédé la place à des systèmes plus complexes (notamment, à plus de trois miroirs ou incluant des correcteurs dioptriques), le qualificatif Cassegrain reste étonnamment moderne et désigne couramment la zone à I'arrière du miroir principal des télescopes actuels. II faut signaler enfin que, pour les systèmes $d$ 'imagerie ou de microscopie, l'objectif Cassegrain constitue la principale

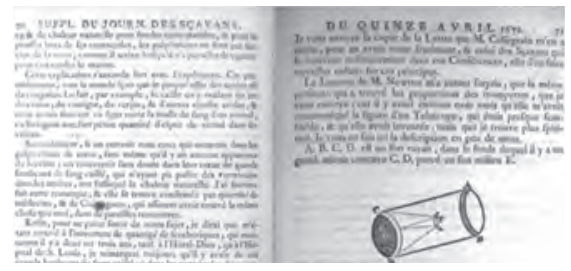

Publication du Sieur de Bercé sur le télescope Cassegrain dans le Supplément du Journal des Sçavans. Source : Bibliothèque de l'Observatoire de Paris, reproduit d'après [1].

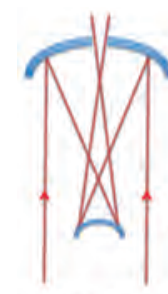

Gregory

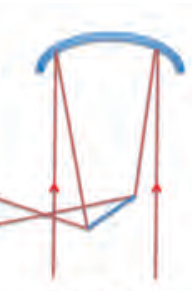

Newton

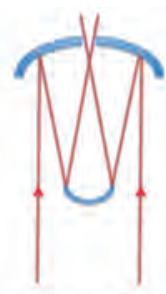

Cassegrain
Schémas de principe des trois grands types de télescopes développés à l'époque de Cassegrain. alternative achromatique aux composants dioptriques standards.

Il n'empêche. Si son nom est connu de tout opticien ou astronome, amateur ou non, Cassegrain lui-même est depuis trois siècles un parfait inconnu. En effet, I'histoire des sciences $n^{\prime}$ a retenu et ne propose que peu d'éléments sur le personnage. Ses seuls écrits identifiés portent sur le mégaphone et le télescope réflectif, tels que publiés dans le supplément au Journal des Sçavans du 15 avril 1672. Jusqu'à il y a peu, les dictionnaires biographiques et autres encyclopédies ne disaient rien de ses origines, sa formation, son œuvre - peinant même à l'identifier formellement et proposant comme seule information Cassegrain, $\mathrm{N}$. Le $\mathrm{N}$., qui a parfois été interprété à tort comme Nicolas, remplace en fait, comme c'est l'usage, le prénom inconnu du physicien...

Ce n'est qu'en 1997 que deux astronomes Français, André Baranne et Françoise Launay [1], se basant sur une information de la Nouvelle Biographie Générale de Ferdinand Hoefer [2] indiquant que Cassegrain enseignait à Chartres, parviennent à retrouver son prénom : Laurent, professeur de seconde au Collège Pocquet. À la même période, un certain Laurent Cassegrain est mentionné comme curé de la paroisse de Chaudon. Baranne et Launay retrouvent et comparent les signatures du curé et du professeur, et parviennent ainsi à remonter la piste du grand homme après 300 ans de silence... 


\section{Éléments de biographie}

Laurent Cassegrain est né dans la région de Chartres vers 1629, dans une France affaiblie et troublée par une sévère épidémie de peste. Il est le fils de Mathurin Cassegrain et Jehanne Marquet (ou Marguet). Mathurin et Jehanne sont connus comme honnêtes homme et femme, ce qui indique à la fois une culture générale étendue et un niveau de vie confortable. Mathurin est marchand mercier (donc à ce titre, membre d'une corporation parisienne spécialisée dans le commerce international des tissus) et épicier à Chartres. II semble avoir aussi fait le commerce de microscopes. La famille Cassegrain jouit d'une certaine notoriété à Chartres, ayant produit plusieurs générations de maîtres-chirurgiens.

Laurent est l'aîné de sa fratrie, avec cinq frères et sœurs tous nés dans la région de Chartres entre 1631 et 1646. On n'a pas encore retrouvé d'informations sur ses jeunes années et sa formation, mais on sait qu'en 1654 il enseigne en seconde au Collège Pocquet de Chartres. II a alors
24 ans. Ses lettres montrent une culture de physicien, avec un intérêt prononcé pour l'acoustique, l'optique et la mécanique. C'est le cas notamment dans les deux articles qu'il soumet en 1672 à l'attention du Sieur de Bercé.

Les registres des églises de Chartres et de Chaudon projettent un peu de lumière sur la vie nimbée de mystère et le caractère de Laurent Cassegrain. Au travers de quelques épisodes de la vie paroissiale (mariage, baptême etc.), comme des flashs stroboscopiques, on y découvre un personnage discret, humble, relativement aisé. Dès 1657, il est présenté comme prêtre. En novembre 1685, il remplace le curé Esme d'Orange qui est nommé chapelain de la Cathédrale de Chartres, et signe son premier acte dans les registres paroissiaux de Chaudon. On peut s'étonner de cette modeste nomination pour celvi qui est, sans doute possible, une personnalité locale, professeur au Collège Pocquet de Chartres, philosophe et savant, en relation suivie avec les hommes de science, I'ami du chanoine Estienne... Sur ce point, le mystère Cassegrain reste entier.
Citons pour finir une anecdote qui montre l'intransigeance morale et la sévérité du prêtre Cassegrain. Louis XIV vient de révoquer l'Edit de Nantes, qui interdit désormais le protestantisme sur le territoire français. Dans cette atmosphère tendue, Laurent Cassegrain s'oppose en février 1689 à l'inhumation en terre bénite d'une protestante qui, bien que récemment convertie, n'a pas suffisamment montré de zèle catholique.

Cassegrain décède à Chaudon, dans la nuit du 31 août 1693. II est inhumé le mercredi 2 septembre au matin dans le cimetière, aujourd'hui disparu, entourant l'église de sa paroisse.

\section{Références}

[1] André Baranne et Françoise Launay, Cassegrain : un célèbre inconnu de l'astronomie instrumentale, J. Opt. 28, pp. 158-172 (1997).

[2] Ferdinand Hoefer, Nouvelle Biographie Générale, Editions Firmin-Didot (Paris, 1855).

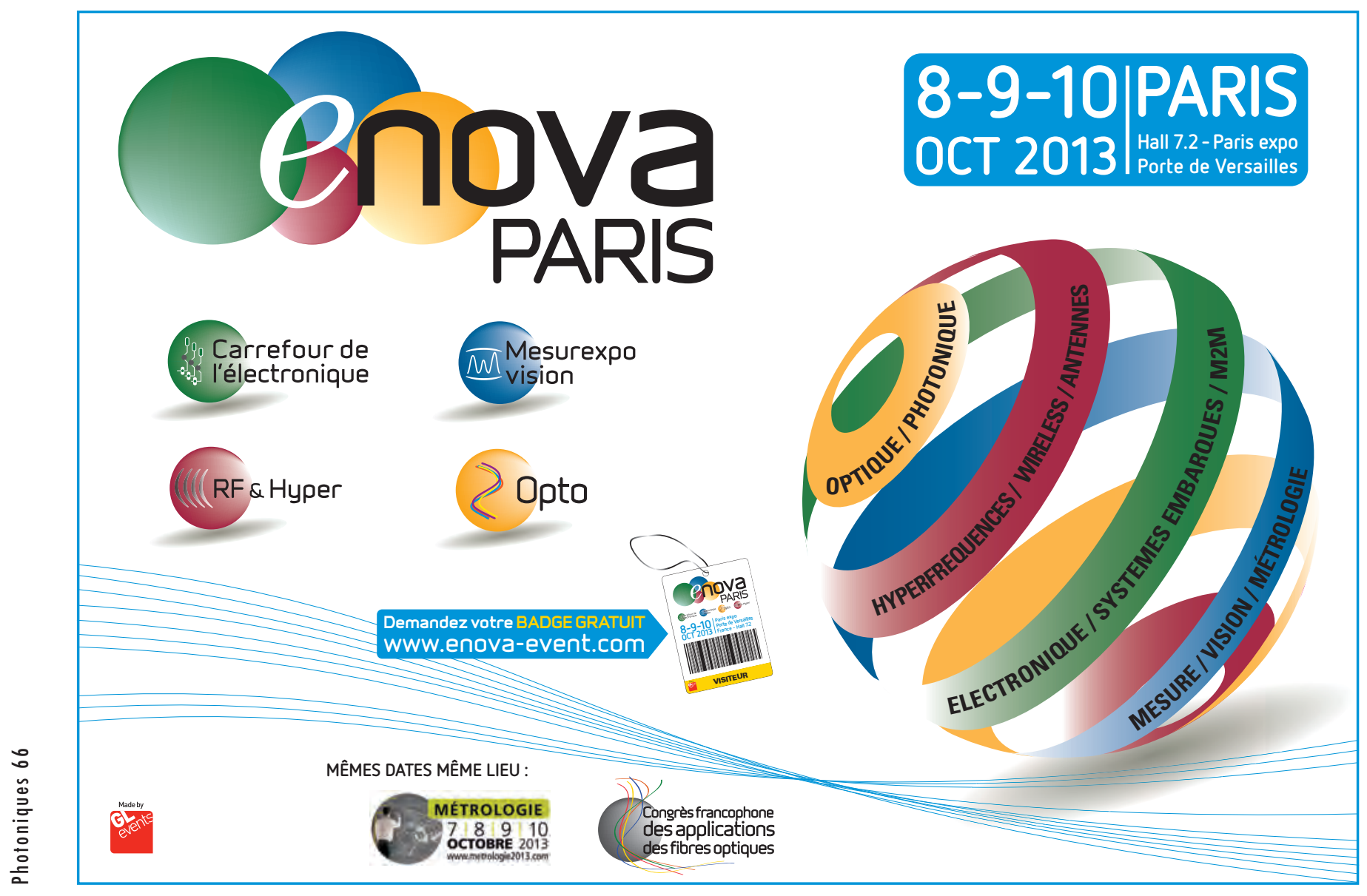

